\title{
SOFIA observations of the planetary nebula NGC 7009
}

\author{
R. H. Rubin ${ }^{1}$, S. W. J. Colgan ${ }^{1}$, R. L. M. Corradi ${ }^{2}$, R. Sankrit ${ }^{3}$, \\ A. G. G. M. Tielens ${ }^{4}$, Y. G. Tsamis ${ }^{5}$, and V. Sivaraja ${ }^{1}$ \\ ${ }^{1} \mathrm{NASA} /$ Ames \\ ${ }^{2}$ Instituto de Astrofísica de Canarias \\ ${ }^{3}$ SOFIA Science Center \\ ${ }^{4}$ Leiden Observatory \\ ${ }^{5} \mathrm{ESO}$
}

\begin{abstract}
We report spectrophotometric observations made with SOFIA/FORCAST on 2011 June 2 UT. Optical measurements have previously shown that the abundance discrepancy factor $(a d f)$ varies with position in several high- $a d f \mathrm{PNe}$, and is highest close to the central star. The very low electron temperature inclusions postulated to explain the abundance discrepancy, must be cooled predominantly by fine structure IR lines. These SOFIA data will map mid-IR FS lines (and our related Herschel program will add several far-IR FS lines) in the bright, wellcharacterized, high- $a d f$ PN NGC 7009. We will compare these IR results with FS optical line measurements in order to correlate ratios of IR to optical fluxes with position, and thus correlate with where the adf peaks.
\end{abstract}

Keywords. ISM: abundances, planetary nebulae: individual (NGC 7009)

\section{Introduction}

The emission line analysis of photoionized nebulae is one of the major tools used to study elemental abundances in our own and other galaxies. Gaseous nebulae are laboratories for understanding physical processes in all emission-line sources and are probes for stellar, galactic and primordial nucleosynthesis. Because of their well defined structures and central ionizing source, PNe provide one of the best laboratories to probe the physical and astrophysical processes that affect the derivation of abundances. It is crucial to have reliable prescriptions for deriving these abundances. However, the present state of affairs continues to contain a long-standing, unsolved mystery. The 'abundance discrepancy problem' refers to this issue, whereby heavy element abundances relative to hydrogen derived from optical recombination lines (ORLs) are generally higher than those obtained from collisionally excited lines (CELs). Often this difference is expressed in terms of the abundance discrepancy factor $(a d f)$, defined as the ratio of the ORLto the CEL-derived value. The adf often exceeds two in $\mathrm{PNe}$ (and $\mathrm{H}$ II regions), and is dramatically high in some PNe: $~ 5$ (NGC 7009), 10 (NGC 6153), 16 (NGC 2022), $\sim 30$ (NGC 1501), up to a factor of $\gtrsim 70$ (Hf 2-2) (Liu 2010 and references therein). Such "extreme" ( $a d f \gtrsim 5)$ nebulae, albeit rare ( $\sim 10 \%$ of PNe surveyed), are nevertheless very important for understanding the underlying physics of the abundance determination problem.

Perhaps the most promising proposition to explain the nebular abundance problem posits that these particular nebulae contain (at least) two distinct emission regions - one of "normal" electron temperature $\left(T_{e}\right) \sim 10000 \mathrm{~K}$, and chemical composition ( $\sim$ solar) and another of very low $T_{e}$ that is H-deficient, thus having high metal abundances relative to 
hydrogen. The latter component emits strong heavy element ORLs and forbidden lines from the lowest-lying energy levels that give rise to IR CELs, but essentially no opti$\mathrm{cal} / U V$ CELs. The consistent picture that emerges from fitting a 2-abundance photoionization model to the spectroscopic data is that the H-poor component is in high-density inclusions, which provide only a minor fraction of the total nebular mass. Yuan et al. (2011) used the code MOCASSIN (Ercolano et al. 2005) to construct a detailed Monte Carlo, 3-dimensional photoionization model of the PN NGC 6153, comparing the model with a broad array of observational data, including HST data. The modeling including the putative low temperature, H-deficient knots does a superior job fitting the data than do models without the clumps. For any of the class of extreme PNe, it has not been possible to spatially resolve one of these knots with ground-based telescopes using stateof-the-art equipment. Tsamis et al. (2008) used the VLT 8.2-m/FLAMES instrument to perform integral-field spectroscopy of NGC $6153\left(0.52^{\prime \prime} \times 0.52^{\prime \prime}\right.$ pixels $)$. The data are consistent with the existence of plasma regions which are both cold and metal-rich, appearing as undulations in the ORL abundance diagnostics of $\sim 1^{\prime \prime}$ across; any associated structures should have physical sizes of up to $\sim 1000$ AU.

\section{How SOFIA observations can help}

With the present SOFIA observations (and a similar Herschel program), we embark upon an alternative observational technique to test the hypothesis of the existence of such inclusions. The picture of two distinct components of highly ionized gas at very different $T_{e}$ could solve the paradox, but the only known way to maintain a strongly photoionized gas at the low $T_{e}(\lesssim 1000 \mathrm{~K})$ expected for the clumps is by fine-structure (FS) IR line cooling. An essential check can be provided by the 2-D spatial distribution of IR FS line emission (Tsamis et al. 2008). Not only does adf vary between objects, but also for several PNe with high adf's, and sufficient angular sizes, the adf is found to correlate with projected distance from the central star, peaking in the inner regions: NGC 6153 (Liu et al. 2000), NGC 6720 (Ring nebula) (Garnett \& Dinerstein 2001) and NGC 2392 (Eskimo nebula) (see discussion p.972 in Tsamis et al. 2004 of Barker 1991 carbon line measurements). The basic idea is that mapping should reveal that the IR FS lines peak at the locations where the adf peaks in the inner regions. This sort of correlative analysis would show that large IR FS gas cooling correlates with high-adf positions. Optical FS lines are negligible at the low $T_{e}$ of the inclusions.

\section{SOFIA observations of NGC $\mathbf{7 0 0 9}$}

On a Basic Science flight 2011 June 2 UT, NGC 7009 was observed on the longest flight leg of the night (156 minutes planned). The preliminary flight plan is shown in Figure 1 with the anticipated ground track; that for NGC 7009 is leg number (16). The entire leg was flown at 43,000 feet $(13,100 \mathrm{~m})$, an advantage due to the lighter load which results from fuel already used. The calibration leg (17) on $\beta$ And was also at 43,000 feet. Observations were made using 6 FORCAST filters: long wavelength channel (LWC) 31.5, 33.6, 34.8, 37.1; short wavelength channel (SWC) 19.7, 24.2. The transmission in these filters along with the positions of the fine structure (FS) emission lines of interest here are shown in Figure 2. These included 3 emission lines - [O IV] 25.9, [S III] 33.5, and [Ne III] $36.0 \mu \mathrm{m}-$ as well as the underlying continuum. We knew from previous observations that [Ne V] 24.3 and [Si II] 34.8, are absent. See the ISO spectrum in Figure 3. The [S III] 18.7 line is expected to contribute much less than the continuum in the 19.7 filter. FORCAST covers a field of view of $3.2^{\prime} \times 3.2^{\prime}$ with $0.75^{\prime \prime}$ pixels, although the theoretical spatial 


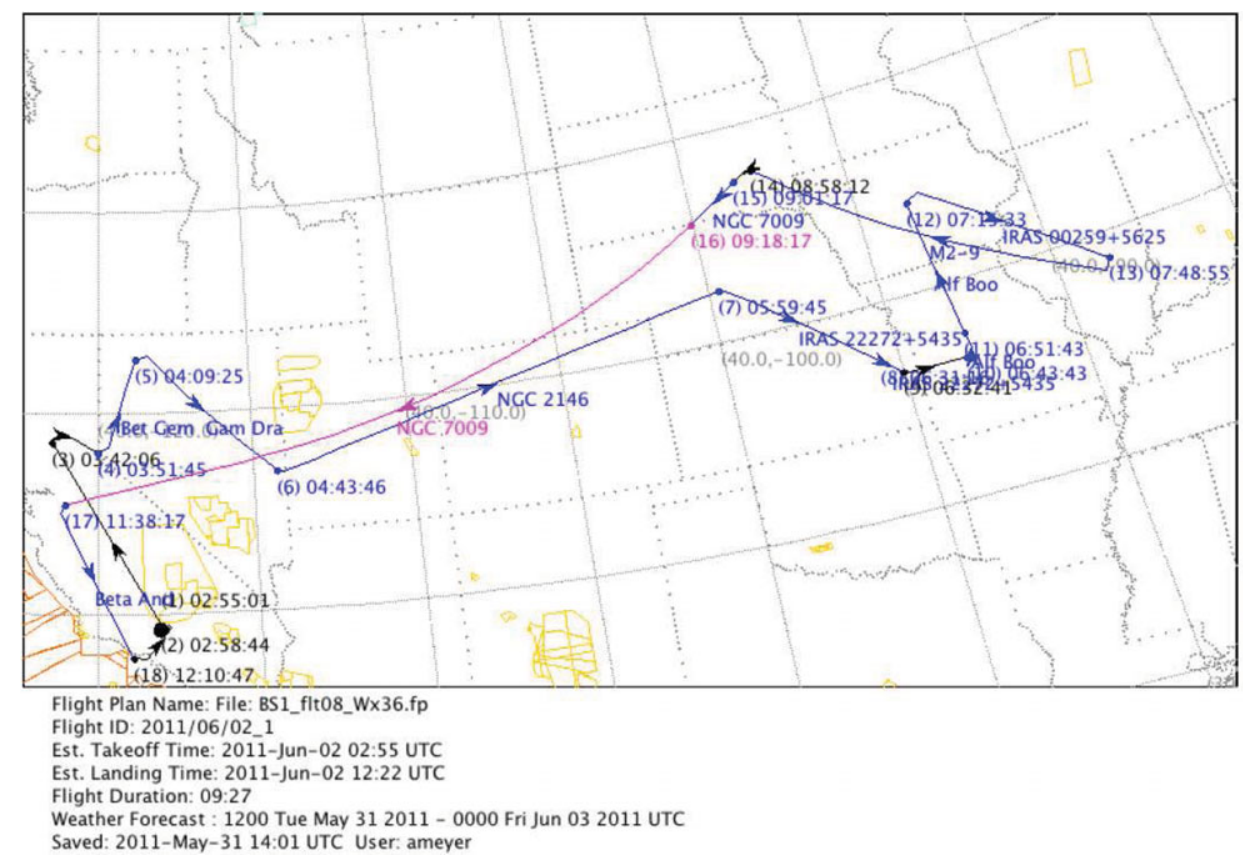

Figure 1. Preliminary flight plan. NGC 7009 was observed for the longest leg and at an altitude of 43,000 feet $(13,100 \mathrm{~m})$.

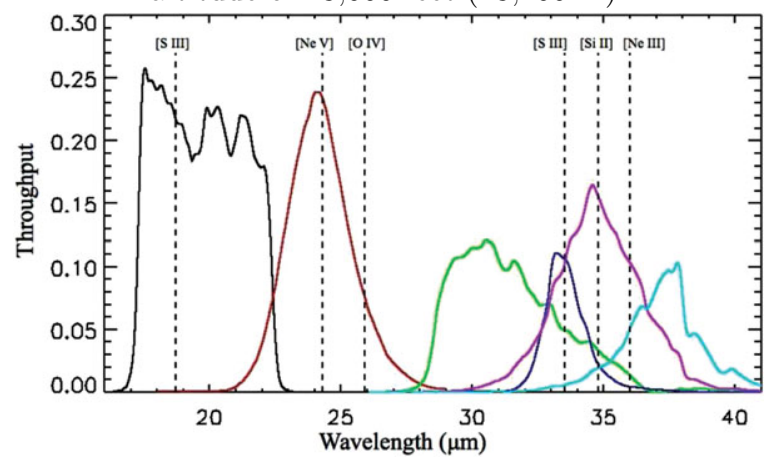

Figure 2. FORCAST filters observed: long wavelength channel (LWC) 31.5, 33.6, 34.8, 37.1; short wavelength channel (SWC) 19.7, 24.2. The positions of the fine structure (FS) emission lines discussed are indicated.

resolution near $35 \mu \mathrm{m}$ is $\sim 3^{\prime \prime}$. As of now, the calibrated pipeline data have not yet been delivered. The resulting 2-D maps in these $3 \mathrm{FS}$ lines of different ions should produce an interesting stratification with the highest ionization species $\mathrm{O}^{3+}$ concentrated closest to the central star. As a prelude, Figure 4 shows the preliminary accumulated data performed in real time onboard the SOFIA flight. One of the panels displays cross cuts along the major and minor axes for the various filters observed.

\section{What we plan to do with the data next}

After the calibrated pipeline data become available, we will tessellate each of the 6 filter maps to 5 pixel $\times 5$ pixel $(\sim 4 \operatorname{arcsec} \times 4$ arcsec $)$ spatial elements. For each spatial tile, we will then solve for 3 line fluxes $-[\mathrm{O}$ IV] 25.9, [S III] 33.5, and [Ne III] $36.0 \mu \mathrm{m}$-as 


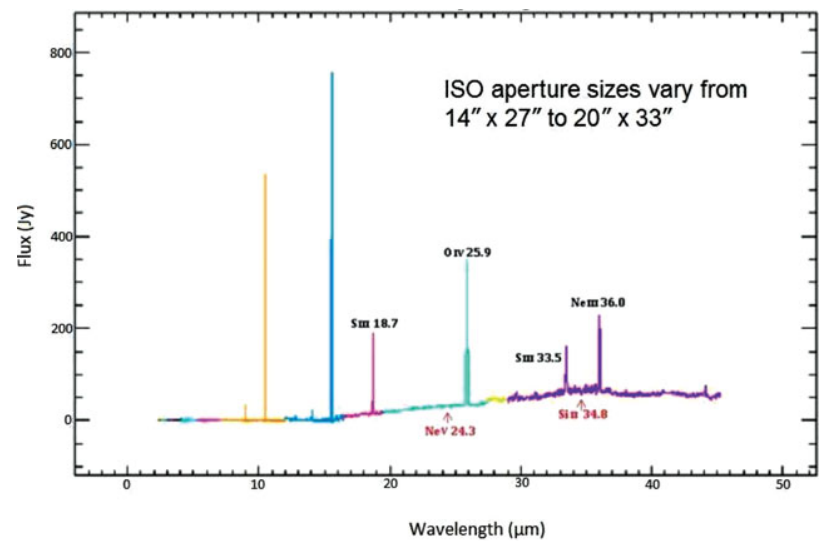

Figure 3. ISO spectrum SWAA73801242 that was taken in high-resolution SWS01 mode.

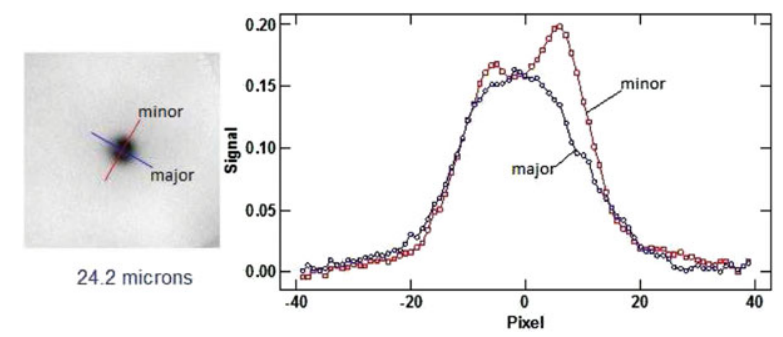

Figure 4. Onboard display of accumulated data for the SWC 24.2 filter that covers the [O IV] $25.9 \mu \mathrm{m}$ line. These cross cuts are along the major and minor axis of NGC 7009.

well as fitting the underlying continuum with a quadratic curve. The 6 filter maps provide the values for 6 linear equations in the 6 quantities to be determined.

Armed with the maps in the three FS IR lines, as well as longer wavelength data from our Herschel program, we will compare these line fluxes with optical/UV CEL fluxes measured at corresponding spatial positions. For instance, there are HST STIS spectra and WFPC2 narrow-band images taken under GO 8114 (PI Rubin). A correlative analysis will be done to test if the flux ratio of IR to optical/UV follows the trend of variations in the $a d f$.

We thank our students Vishal Kalyanasundaram, Zeyu Liu, Zezhou Liu, and Scott Zhuge for their considerable help with this project. RR is grateful for funding from the SOFIA Science Center.

\section{References}

Barker, T. 1991, ApJ, 371, 217

Ercolano, B., Barlow, M. J., \& Storey, P. J. 2005, MNRAS, 362, 1038

Garnett, D. R., \& Dinerstein, H. L. 2001,ApJ, 558, 145

Kingsburgh, R. L., \& Barlow, M. J. 1994, MNRAS, 271, 257

Liu, X-W. 2010 (astro-ph/1001.3715)

Liu, X.-W., et al. 2000, MNRAS, 312, 585

Tsamis, Y. G., Barlow, M. J., Liu, X.-W., Storey, P. J., \& Danziger, I. J. 2004, MNRAS, 353, 953

Tsamis, Y. G., et al. 2008, MNRAS, 386, 22

Yuan, H.-B., et al. 2011, MNRAS, 411, 1035 